\title{
Impact of a Formulation Containing Unusual Polyunsaturated Fatty Acids, Trace Elements, Polyphenols and Plant Sterols on Insulin Resistance and Associated Disturbances
}

\author{
María J. Peláez-Jaramillo • Natalia Valencia-Enciso • Allison A. Cárdenas-Mojica • Paula V. Gaete • \\ Eitan A. Scher-Nemirovsky • Luisa F. Gómez-Arango · Daniel Colmenares-Araque • \\ Camilo A. Castro-López • Eddy Betancourt-Villamizar · Jesús Jaimes-Madrigal • Carlos A. Alvarez • \\ Mario A. Jiménez-Mora · Pedro J. Quiroga-Padilla · Danna K. Puerto-Baracaldo · Carlos O. Mendivil (D)
}

Received: October 14, 2019 / Published online: November 5, 2019

(C) The Author(s) 2019

\section{ABSTRACT}

Introduction: To evaluate the effect of a lipidbased formulation containing unusual polyunsaturated fatty acids, trace elements, polyphenols and plant sterols on insulin resistance and its associated disturbances among adults at risk of diabetes.

Methods: This was an 8-week, three-arm, openlabel randomized clinical trial. We studied individuals aged $\geq 18$ years old with diabetes risk given by a body mass index $\geq 25 \mathrm{~kg} / \mathrm{m}^{2}$ or a

Enhanced Digital Features To view enhanced digital features for this article go to: https://doi.org/10.6084/ m9.figshare.10033112.

M. J. Peláez-Jaramillo · N. Valencia-Enciso ·

A. A. Cárdenas-Mojica · P. V. Gaete .

E. A. Scher-Nemirovsky · M. A. Jiménez-Mora ·

P. J. Quiroga-Padilla . D. K. Puerto-Baracaldo .

C. O. Mendivil $(\varangle)$

School of Medicine, Universidad de los Andes, Bogotá, Colombia

e-mail: cmendivi@uniandes.edu.co

L. F. Gómez-Arango - D. Colmenares-Araque .

C. A. Castro-López · E. Betancourt-Villamizar .

J. Jaimes-Madrigal · C. A. Alvarez

Team Foods Colombia, S.A., Bogotá, Colombia

C. O. Mendivil

Section of Endocrinology, Department of Internal

Medicine, Fundación Santa Fe de Bogotá, Bogotá,

Colombia
FinnRisc score $\geq 13 / 20$. Participants were randomly assigned to receive: $7 \mathrm{ml}$ sunflower oil (control group), $3.5 \mathrm{ml}$ of the study formulation $+3.5 \mathrm{ml}$ of sunflower oil (low-dose group) or $7 \mathrm{ml}$ of study formulation (high-dose group). Results: We randomized 25 individuals. After one withdrawal in the high-dose group, the study sample comprised nine patients in the control, nine in the low-dose and six in the high-dose groups. The insulin sensitivity increased significantly and in a dose-dependent fashion, up to $10 \%$ in the high-dose group. At week 8 the low-dose group exhibited lower glycemic excursions during the oral glucose tolerance test (OGTT), especially $1 \mathrm{~h}$ after the glucose challenge $(32 \mathrm{mg} / \mathrm{dl}$ or $23 \%$ lower vs. control group). The incremental area under the glucose curve in the OGTT was $17.1 \%$ lower in the low-dose group vs. the control group. Waist circumference increased in the control group, remained constant in the low-dose group and decreased in the high-dose group. C-reactive protein decreased in both formulation groups, up to $50 \%$ in the high-dose group. Participants in the formulation groups exhibited increased secretion of GLP-1 and plasma irisin at week 8 vs. the control group.

Conclusion: The formulation induced favorable changes in insulin sensitivity, glucose tolerance, abdominal obesity and inflammation. 
These effects and their durability will need to be assessed in larger studies.

Trial Registration: NCT03512665.

Funding: Team Foods Colombia.

Keywords: Adipokines; Diabetes; Fatty acids; Gastrointestinal hormones; Insulin resistance; Metabolic syndrome

\section{Key Summary Points}

Why carry out this study?

Insulin resistance and prediabetes are highly prevalent, particularly among Hispanic/Latino populations, and carry a host of serious long-term complications.

A formulation combining several components with nutraceutical properties is likely to positively impact insulin resistance and its associated disturbances among adults at risk of diabetes.

\section{What was learned from the study?}

The lipid-based formulation evaluated in the study showed a positive effect on insulin resistance and its associated disturbances and hormonal profile.

The study intervention induced favorable changes in insulin sensitivity, glucose tolerance, abdominal obesity, inflammation and peptide hormones related to nutrient metabolism.

\section{INTRODUCTION}

Non-communicable diseases (NCDs) are the primary cause of mortality worldwide, accounting for $73.4 \%$ of deaths in 2017 [1]. The past 10 years have witnessed a $22.7 \%$ increase in mortality due to cardiovascular diseases, chronic respiratory diseases, diabetes, kidney diseases and neoplasms, among others [1]. Sixty-five percent of deaths attributable to risk factors are due to NCDs, representing 26.6 million deaths per year [2]. Metabolic risk factors are a pivotal determinant of the increasing burden of NCDs and show a worrisome upward trend in low- and middle-income countries [2].

Prediabetes, characterized by elevated plasma glucose without crossing diagnostic thresholds for diabetes, is the first consequence of insulin resistance and a major risk factor for developing clinical diabetes and its complications. The term prediabetes encompasses impaired fasting glucose (IFG) and impaired glucose tolerance (IGT). Insulin resistance is a pathophysiologic precursor to both conditions: IFG is mostly related to reduced hepatic insulin sensitivity, while IGT correlates more with reduced sensitivity at muscle and adipose tissue [3]. Insulin resistance is also accompanied by intracellular lipid accumulation in muscle and liver, and by increased circulating free fatty acids, all of which lead to a chronic proinflammatory state [4]. Additionally, prediabetes is associated with increased cardiovascular risk, microangiopathy, hypertension, obstructive sleep apnea and even cognitive dysfunction [5]. Insulin resistance and its correlates have been associated with the development of cardiovascular disease [6].

Nonpharmacologic interventions in addition to diet and exercise may be useful tools to prevent the negative consequences of insulin resistance. In the USA, people tend to use vitamins and supplements along with their prescribed medicines; in fact, about $18 \%$ of the population substitutes medications with supplements [7]. The most frequently reported reasons for preferring supplements are cost and personal beliefs about their benefits [7]. A large proportion of the general public perceives nonpharmacologic alternatives as more holistic [8], safer and more effective than medications [9].

In this context, we developed a lipid-based formulation that contains a mixture of ingredients with the potential to improve insulin resistance and ameliorate the metabolic disturbances associated with it. The ingredients of the formulation can be grouped as follows: unusual polyunsaturated fatty acids, trace elements, polyphenols and plant sterols. The formulation has been submitted for intellectual property protection under the provisional patent application no. US 62/746991. 


\section{Unusual Polyunsaturated Fatty Acids}

Punicic acid is an 18-carbon, polyunsaturated fatty acid (18:3 n-5), usually grouped with other fatty acids collectively considered "conjugated linolenic acid" [10]. In C57Bl/J6 mice, supplementation of the diet with just $1 \%$ punicic acidrich oil (pomegranate), prevented excessive adiposity and improved whole-body insulin sensitivity [11]. Similarly, in a different animal model [the OLETF rat model of human type 2 diabetes mellitus (T2DM)], dietary supplementation with punicic acid prevented the development of hepatic steatosis, a frequent comorbidity of obesity and the metabolic syndrome [12].

Palmitoleic acid is a monounsaturated, 16-carbon fatty acid (16:1, n-7), which functions more as a signaling molecule or lipokine than as an energy source [13]. Plasma concentrations of palmitoleic acid are negatively associated with risk of T2DM and hypertriglyceridemia in humans [14]. Several animal studies [15] and one randomized clinical trial in human adults [16] have documented positive effects of dietary supplementation with cis-palmitoleic acid on plasma triglycerides, LDL cholesterol and HDL cholesterol.

Pinolenic acid is an isomer of gamma-linolenic acid, consisting of 18 carbons with 3 double bonds starting in the $\mathrm{n}-6$ position (18:3, $\mathrm{n}-6)$. The difference lies in the position of the third double bond, which is located at position $\mathrm{n}-12$ in gamma-linolenic and in position n-13 in pinolenic acid. Consumption of pinolenic acid is accompanied by significant acute increases in the appetite-curbing gastrointestinal hormones glucagon-like peptide 1 (GLP-1) and cholecystokinin-8 (CCK-8) in postmenopausal women [17].

\section{Trace Elements}

Chromium is a trace element in the human diet, with a relevant role in insulin signaling and the potential to improve insulin sensitivity and influence nutrient metabolism [18]. Clinical studies in patients with newly diagnosed T2DM found that dietary supplementation with $42 \mu \mathrm{g}$ chromium (as $9 \mathrm{~g}$ of brewer's yeast) over
3 months generated significant reductions of plasma glucose and glycated hemoglobin (HbA1c), with a concomitant positive impact on plasma lipids [19]. However, studies in patients with longer-duration T2DM have not found a positive effect of chromium supplementation [20]. Mechanistically, chromium supplementation appears to reduce plasma levels of proinflammatory cytokines and oxidative stress [21].

\section{Polyphenols}

Curcumin is a polyphenolic compound extracted from the roots of the plant Curcuma longa (turmeric), which is used as both a condiment and a natural medicine in many cultures worldwide [22]. A prior 3-month trial in patients with type 2 diabetes (T2DM) found that dietary supplementation with $300 \mathrm{mg} /$ day of curcuminoids led to a $28 \%$ decrease in the Homeostasis Model Assessment-Insulin Resistance (HOMA-IR) index with a concomitant reduction of $0.7 \%$ points in $\mathrm{HbA} 1 \mathrm{c}$ [23]. Other trials restricted to patients with obesity have documented a positive impact of the same dose of curcuminoids on plasma triglycerides [24].

\section{Plant Sterols}

Finally, plant sterols are compounds that compete with dietary cholesterol for intestinal transport and thus may have a positive impact on plasma lipids in patients with metabolic syndrome and/or dyslipidemia [25].

The aim of this study was to perform an initial evaluation of the effect of a lipid-based formulation containing punicic acid, palmitoleic acid, pinolenic acid, chromium, curcumin and plant sterols on insulin resistance and associated disturbances among adults at risk of T2DM.

\section{METHODS}

\section{Study Design}

This was an 8-week, three-arm, open-label randomized clinical trial. 


\section{Patients}

Inclusion criteria for study participants were age $>18$ years (men and women), T2DM risk given by a body mass index $\geq 25 \mathrm{~kg} / \mathrm{m}^{2}$ or a FinnRisc score $\geq 13 / 20$, and willingness to keep diet and physical activity habits constant throughout participation in the study. FinnRisc is a screening tool for T2DM risk based on the Finnish Diabetes Prevention Study, which has been extensively validated in Hispanic populations, including a direct validation in Colombia [26].

Exclusion criteria were to be involved in an active weight-loss program, to be receiving any medication with potential effect on insulin resistance or metabolic parameters (with the exception of oral contraceptives or levothyroxine, which had to be received at the same dose since at least 6 months prior), hypersensitivity to any of the components of the study formula, gastrointestinal or other health conditions that might compromise compliance with the study interventions, known diabetes or diabetes complications, alcohol or drug abuse, pregnancy and lactation. Potential participants were recruited between February 2018 and June 2018 from a database of volunteers that we keep at the Nutritional Biochemistry Laboratory at Universidad de los Andes and have been maintaining and curating over the last few years.

Potential participants were sent to a screening appointment where they filled out a selection criteria questionnaire, and their weight, height and waist circumference were measured and their FinnRisc score calculated. Patients who fulfilled the selection criteria were later contacted for their week 0 visit, in which we obtained written informed consent, completed their baseline evaluation, handed them the study intervention for the first 4 weeks and provided them with a compliance diary.

\section{Sample Size}

Assuming a coefficient of variation of $20 \%$ for the insulin sensitivity index (ISI-Gutt) among individuals at risk of T2DM [27], a sample size of 21 participants ( 7 in the control group, 7 in the low-dose formulation group and 7 in the highdose formulation group) would give us $80 \%$ power to detect a true difference of at least 30\% in the baseline-subtracted change in ISI-Gutt between any of the intervention groups relative to the control group at a two-tailed significance level of 0.1 . Foreseeing a $20 \%$ loss to follow-up rate, we recruited 25 patients in total.

\section{Baseline and Follow-Up Evaluations}

We performed the following assessments in all participants at week 0 and at week 8 (end of follow-up): (1) anthropometry and body composition by impedanciometry, (2) a food frequency questionnaire, previously developed for the Colombian population [28], (3) the short version of the international Physical Activity Questionnaire (iPAQ) [29], (4) clinical chemistry including HbA1c, a lipid panel, oral glucose tolerance test (OGTT), liver transaminases, gamma-glutamyl transferase (GGT), high-sensitivity C-reactive protein (hsCRP), plasma creatinine with estimated glomerular filtration rate (eGFR) and urinary albumin excretion, (5) plasma concentrations of metabolic hormones including insulin, glucagon-like peptide-1 (GLP-1), ghrelin, fibroblast growth factor-21 (FGF-21) and irisin. All participants had one additional visit at week 4 , in which we measured anthropometry, body composition and blood pressure, collected unused intervention flasks and documented adherence and adverse events.

\section{Randomization Procedure}

Between the screening visit and the week 0 visit, participants were randomly assigned to an intervention group by means of a random number generator; the random number was between 0 and 1 . Participants assigned a number $<0.33$ were assigned to the control group, participants with numbers $\geq 0.33$ but $<0.67$ were assigned to the low-dose formulation group, and participants assigned a number $\geq 0.67$ were assigned to the high-dose formulation group. 


\section{Study Interventions}

The three study groups received respectively:

- Control group $7 \mathrm{ml}$ of sunflower oil (selected for being rich in polyunsaturated fatty acids and hence a healthy type of oil).

- Low-dose formulation group $3.5 \mathrm{ml}$ of study formulation $+3.5 \mathrm{ml}$ of sunflower oil (previously mixed in the same flask).

- High-dose formulation group $7 \mathrm{ml}$ of the study formulation, containing punicic acid-rich oil (pomegranate seed oil), palmitoleic acid-rich oil (buckthorn oil), pinolenic acid-rich oil (pine nut oil), curcumin, chromium picolinate and campesterol (a plant sterol).

The intervention was meant to be taken in a single take right after breakfast, once a day, over the 8 weeks of the study (56 doses per participant). All participants were asked to maintain their usual dietary and physical activity habits as constant as possible during the duration of the study. The typical Colombian diet has very low amounts of the compounds in the study formulation. The study interventions were packed in individual twist-off flasks (one dose per flask); 28 flasks were packed in a small portable cardboard box that had a compliance diary printed in it, so that participants could cross off every dose they took. Participants were also asked to return any unused flasks to better assess adherence. At weeks 4 and 8, participants responded to a questionnaire on adverse events that asked about their presence, nature, severity, duration and whether participants associated them with the intake of the study intervention.

\section{Laboratory Measurements}

Most clinical chemistry determinations were done in duplicate using colorimetric kits from Wiener Laboratory, Argentina, and Reflotron ${ }^{\circledR}$ clinical chemistry test strips (Hoffman-La Roche, Switzerland). High-sensitivity C-reactive protein (hsCRP) was measured using an immunoturbidimetric latex assay from BioSystems (CAT\# 22921, BioSystems, Spain). HbA1c and urinary albumin excretion were measured employing immunocolorimetric kits from NycoCard (AxisShield, Norway); catalog numbers of the individual and/or multiplex ELISA kits employed for the determinations of plasma concentrations of hormones are respectively: insulin: Abcam CAT\# ab213815; GLP-1 and ghrelin: Millipore Magpix CAT\# HMHEMAG34K05; FGF-21: Abcam CAT\# ab125966 and irisin: Phoenix CAT\# EK-067-29.

\section{Statistical Analysis}

Our main study outcome was the baseline-subtracted between-group difference in the ISI-Gutt in each intervention group compared with the control group. With exploratory intention, we also analyzed the between-group differences in anthropometric measures, area under the glucose curve in the OGTT, HbA1c, lipid variables and plasma concentrations of metabolic hormones. Differences in numeric variables between each treatment group and the control group were analyzed employing non-parametric Mann-Whitney $U$ tests. Between-group comparisons of categorical variables were done with chi-squared tests with continuity correction. All analyses were done at a nominal significance value of 0.05 .

\section{Ethical Aspects}

The study was approved by a legally constituted external Internal Review Board (Riesgo de Fractura, SAS), according to minute 30194 of 2018. All patients underwent an extensive and detailed informed consent procedure and provided written informed consent for their participation. All study procedures were executed in compliance with scientific, technical and administrative regulations for health research dictated by resolution 008430-1993 of the Colombian Ministry of Health and with the principles stated by the Helsinki Declaration of 1964 and its later amendments. The study is registered in the public registry of clinicaltrials.gov under number NCT03512665. 
Table 1 Baseline characteristics of study participants

\begin{tabular}{|c|c|c|c|c|}
\hline & Control & Low dose & High dose & $p$ value \\
\hline Age (years) & $35.7(17.3)$ & $28.9(11)$ & $34.9(19.8)$ & 0.63 \\
\hline Weight (kg) & $78.7(13.6)$ & $78.8(13.4)$ & $74.6(13)$ & 0.78 \\
\hline Height $(\mathrm{cm})$ & $166.3(9.1)$ & $166.3(10.2)$ & $160.4(8.3)$ & 0.38 \\
\hline Body mass index $\left(\mathrm{kg} / \mathrm{m}^{2}\right)$ & $28.4(3.7)$ & $28.3(3.3)$ & $28.8(2.8)$ & 0.95 \\
\hline$\%$ Body fat & $34.8(9.1)$ & $28.3(3.3)$ & $28.8(2.8)$ & 0.35 \\
\hline Waist circumference $(\mathrm{cm})$ & $90(12.7)$ & $95.4(12)$ & $96(9.1)$ & 0.51 \\
\hline Muscle mass (kg) & $48.9(10.2)$ & $50.4(11.4)$ & $43.1(4.7)$ & 0.31 \\
\hline Systolic blood pressure $(\mathrm{mmHg})$ & $121.9(13.8)$ & $113.3(13.7)$ & $114.9(19)$ & 0.47 \\
\hline Diastolic blood pressure $(\mathrm{mmHg})$ & $76(12.3)$ & $71.8(7.9)$ & $69.4(12.3)$ & 0.48 \\
\hline HbAlc (\%) & $5.2(0.5)$ & $5.2(0.5)$ & $5.0(0.4)$ & 0.70 \\
\hline Total cholesterol (mg/dl) & $210.6(51.3)$ & $195.5(23.5)$ & $192.9(43.8)$ & 0.63 \\
\hline HDL cholesterol $(\mathrm{mg} / \mathrm{dl})$ & $39.1(11.8)$ & $35.0(9.3)$ & $50.3(21.2)$ & 0.11 \\
\hline Triglycerides (mg/dl) & $145.4(36.4)$ & $131.3(33.6)$ & $144.7(36.1)$ & 0.65 \\
\hline LDL cholesterol $(\mathrm{mg} / \mathrm{dl})$ & $142.4(46.1)$ & $134.2(22.8)$ & $113.6(41.6)$ & 0.32 \\
\hline Fasting plasma glucose $(\mathrm{mg} / \mathrm{dl})$ & $91.3(11.7)$ & $87(3.3)$ & $87(6.6)$ & 0.45 \\
\hline AST (UI/l) & $17.0(3.9)$ & $21.3(8.7)$ & $20.1(15.1)$ & 0.62 \\
\hline $\operatorname{ALT}(\mathrm{UI} / \mathrm{l})$ & $20.3(9.8)$ & $23.6(13)$ & $14.0(3)$ & 0.22 \\
\hline GGT (UI/l) & $20.9(21.7)$ & $27.8(23.1)$ & $21.8(17.9)$ & 0.79 \\
\hline Creatininemia (mg/dl) & $0.90(0.13)$ & $0.92(0.15)$ & $0.85(0.06)$ & 0.53 \\
\hline eGFR (ml/min) & $99.2(40.0)$ & $111.9(41.0)$ & $77.5(12.9)$ & 0.21 \\
\hline Urinary albumin/creatinine ratio & $8.5(11.0)$ & $3.2(2.5)$ & $8.8(8.3)$ & 0.30 \\
\hline C-reactive protein $(\mathrm{mg} / \mathrm{l})$ & $2.2(1.7)$ & $2.0(1.2)$ & $3.1(2.8)$ & 0.83 \\
\hline Intense physical activity (min/week) & $23.3(36.1)$ & $35.6(32.4)$ & $8.6(22.7)$ & 0.25 \\
\hline Moderate physical activity (min/week) & $40.6(58.3)$ & $7.2(11.5)$ & $17.1(26.3)$ & 0.19 \\
\hline Mild physical activity (min/week) & $56.1(55.7)$ & $27.8(17.3)$ & $35(30.4)$ & 0.29 \\
\hline Sedentary time (min/week) & $412.5(179.8)$ & $373.3(152.3)$ & $462.9(253.9)$ & 0.66 \\
\hline \multicolumn{5}{|l|}{ Dietary intake } \\
\hline Energy (cal/kg/day) & $35.2 \pm 14.9$ & $31.4 \pm 19.6$ & $26.6 \pm 6.8$ & 0.41 \\
\hline Protein (\% of daily energy) & $13.8(3.1)$ & $16(5.6)$ & $14.4(5)$ & 0.60 \\
\hline Lipids (\% of daily energy) & $36.7(5.9)$ & $37.8(9.4)$ & $30.4(4.7)$ & 0.15 \\
\hline Carbohydrates (\% of daily energy) & $49.5(7.7)$ & $46.3(13.6)$ & $55.3(9.4)$ & 0.30 \\
\hline Fiber (g/day) & $32.2(18.7)$ & $23.6(18.2)$ & $21.3(6.4)$ & 0.39 \\
\hline Calcium (mg/day) & $907(340)$ & $990(807)$ & $929(485)$ & 0.95 \\
\hline
\end{tabular}


Table 1 continued

\begin{tabular}{lllll}
\hline & Control & Low dose & High dose & $\boldsymbol{p}$ value \\
\hline Iron (mg/day) & $19.9(9.8)$ & $19.1(11.1)$ & $16.3(4.7)$ & 0.76 \\
Sodium (mg/day) & $3959(2009)$ & $3248(1949)$ & $2986(1008)$ & 0.55 \\
Phosphorus (mg/day) & $1316(451)$ & $1287(982)$ & $1110(244)$ & 0.83 \\
Zinc (mg/day) & $11.6(3.3)$ & $11(7.2)$ & $9.7(2.3)$ & 0.79 \\
Potassium (mg/day) & $3714(1932)$ & $3296(2587)$ & $2902(618)$ & 0.75 \\
Saturated fats (\% of daily energy) & $17.5(4.9)$ & $18.8(5.9)$ & $16(1.9)$ & 0.56 \\
Monounsaturated fats (\% of daily energy) & $3.1(1.7)$ & $4.1(2.8)$ & $3.4(1.5)$ & 0.61 \\
Polyunsaturated fats (\% of daily energy) & $2.0(1.4)$ & $2.6(1.8)$ & $2.4(1.5)$ & 0.71 \\
Cholesterol (mg/day) & $389(266)$ & $467(418)$ & $358(112)$ & 0.78 \\
Trans fatty acids (g/day) & $1.4(0.7)$ & $1.5(1.3)$ & $0.9(0.5)$ & 0.45 \\
Eicosapentaenoic acid (mg/day) & $31(39)$ & $170(274)$ & $193(269)$ & 0.28 \\
Docosahexaenoic acid (mg/day) & $153(202)$ & $1010(1406)$ & $1079(1503)$ & 0.21 \\
Vitamin A (mcg retinol equivalents/day) & $1077(840)$ & $1079(805)$ & $815(389)$ & 0.76 \\
Vitamin E (mg/day) & $15.1(8.3)$ & $14.2(13.3)$ & $12.4(5.6)$ & 0.88 \\
Vitamin K (mg/day) & $169(125)$ & $93(77)$ & $91(41)$ & 0.17 \\
Vitamin D (mcg/day) & $259(191)$ & $287(210)$ & $229(87)$ & 0.83 \\
Thiamine (mg/day) & $3.1(1.9)$ & $2.8(1.9)$ & $2.2(1)$ & 0.63 \\
Riboflavin (mg/day) & $2.1(1.1)$ & $2.2(1.8)$ & $1.7(0.4)$ & 0.71 \\
Niacin (mg/day) & $43.1(18)$ & $48.6(42.6)$ & $36.9(9.7)$ & 0.74 \\
Vitamin C (mg/day) & $146(98)$ & $121(112)$ & $117(57)$ & 0.80 \\
Folate (mg/day) & $524(235)$ & $410(268)$ & $413(106)$ & 0.50 \\
\hline Data & & & \\
\hline
\end{tabular}

Data are means $(\mathrm{SD})$

$A S T$ aspartate aminotransferase, $A L T$ alanine aminotransferase, $G G T$ gamma-glutamyl transpeptidase, $e G F R$ estimated glomerular filtration rate

\section{RESULTS}

We screened 66 potential participants to find 25 eligible individuals. After randomization, nine participants were allocated to the control group, nine to the low-dose group and seven to the high-dose group. We only had one withdrawal in the high-dose group at week 2 (the participant did not like the taste of the study formulation), so the study sample comprised 24 participants, 9 in the control group, 9 in the low-dose group and 6 in the high-dose group.
Study participants were on average 34 years old, overweight (average BMI 28.6), abdominally obese (average waist circumference $92.7 \mathrm{~cm}$ in women, $96.8 \mathrm{~cm}$ in men) and had a high percentage of body fat (35.4\%). Average blood pressure levels were normal, as were average fasting plasma glucose, HbA1c, plasma lipid, liver enzyme and renal function values. C-reactive protein was slightly elevated, indicating chronic low-grade inflammation. All demographic, clinical and laboratory variables, as well as the average intake of all the analyzed 


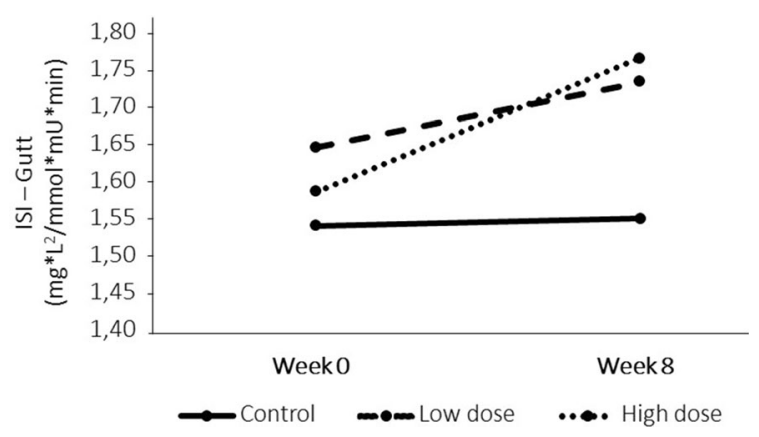

Fig. 1 Change in the insulin sensitivity index by treatment group

macro- and micronutrients, were comparable across the three groups (Table 1).

\section{Insulin Sensitivity and Glucose Tolerance}

The ISI-Gutt employs data from a multipoint OGTT to estimate the amount of whole-body glucose uptake induced by each mUI of insulin, per minute, per kg body weight. ISI-Gutt has been widely validated versus the euglycemic clamp as a reliable index of insulin sensitivity [27]. ISIGutt improved in the two intervention groups (5.4\% in the low-dose and $10.1 \%$ in the highdose group), while in the control group it remained stable (Fig. 1). The $p$ value for the comparison of change in ISI-Gutt in the low-dose vs. control group was 0.34 . For the high-dose vs. control group comparison, the $p$ value was 0.041 .

The study formulation modulated the response to a glucose load in the OGTT, with the most pronounced change observed in the low-dose group. Glycemic levels $60 \mathrm{~min}$ postload were reduced by $32 \mathrm{mg} / \mathrm{dl}$ relative to the

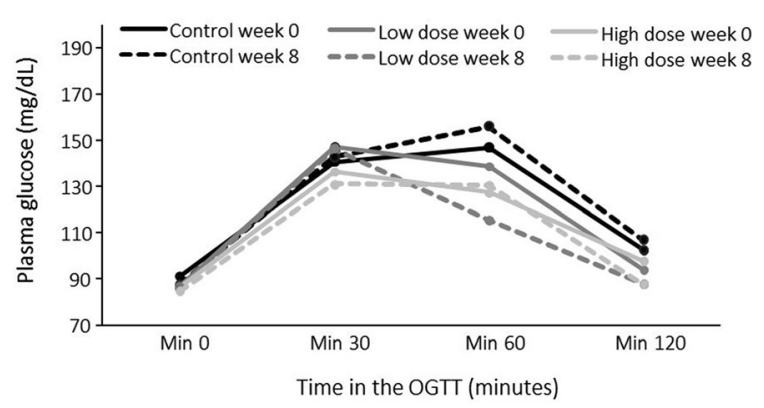

Fig. 2 Glycemic excursions during the oral glucose tolerance test (OGTT) by treatment group control group ( $p=0.047$, Fig. 2). The incremental area under the glucose curve by week 8 was $17.1 \%$ lower in the low-dose group compared with the control group (220.3 vs. $182.6 \mathrm{mg} / \mathrm{dl} \times \mathrm{h}, \quad p=0.073)$. Concerning HbA1c, we observed reductions in the three study groups, with the largest drop occurring in the high-dose group $(-0.36 \%$ in control group, $-0.14 \%$ in low-dose group, $-0.50 \%$ in highdose group) (Fig. 3). However, this difference was not statistically significant.

\section{Other Metabolic Parameters}

Body weight, percent body fat and muscle mass remained relatively constant in the three intervention groups, without differences among them (Table 2). Meanwhile, waist circumference increased slightly in the control group, remained constant in the low-dose group and decreased in the high-dose group (low-dose vs. control $p=0.22$, high-dose vs. control $p=0.012$ ). Changes in HDL cholesterol and triglycerides were small and did not differ among treatment groups. All three study groups showed favorable modifications of LDL cholesterol, but there were no differences in the change in either of the formulation groups vs. the control group (Fig. 3). Plasma aspartate amino transferase (AST) remained constant in the control group but decreased in the formulation groups $(-1.1 \mathrm{U} / \mathrm{l}$ in the control group, $-7.4 \mathrm{U} / 1$ in the low-dose group, $-8.0 \mathrm{U} / \mathrm{l}$ in the high-dose group). Changes in alanine amino transferase (ALT) and gamma-glutamyl transpeptidase (GGT) did not differ across groups (Table 2). The urinary albu$\mathrm{min} /$ creatinine ratio decreased in all three groups (up to $5.0 \mathrm{mg} / \mathrm{g}$ in the high-dose group). Highsensitivity C-reactive protein increased mildly in the control group $(+1.0 \mathrm{mg} / \mathrm{l})$, while it decreased in the two intervention groups $(-0.8 \mathrm{mg} / \mathrm{l}$ in the low-dose group, $p=0.07$ vs. control; $-2.1 \mathrm{mg} / \mathrm{l}$ in the high dose group, $p=0.071$ ) (Table 2 and Fig. 4).

\section{Metabolic Hormones}

Ghrelin is an appetite-inducing peptide hormone, whose production is induced by fasting 

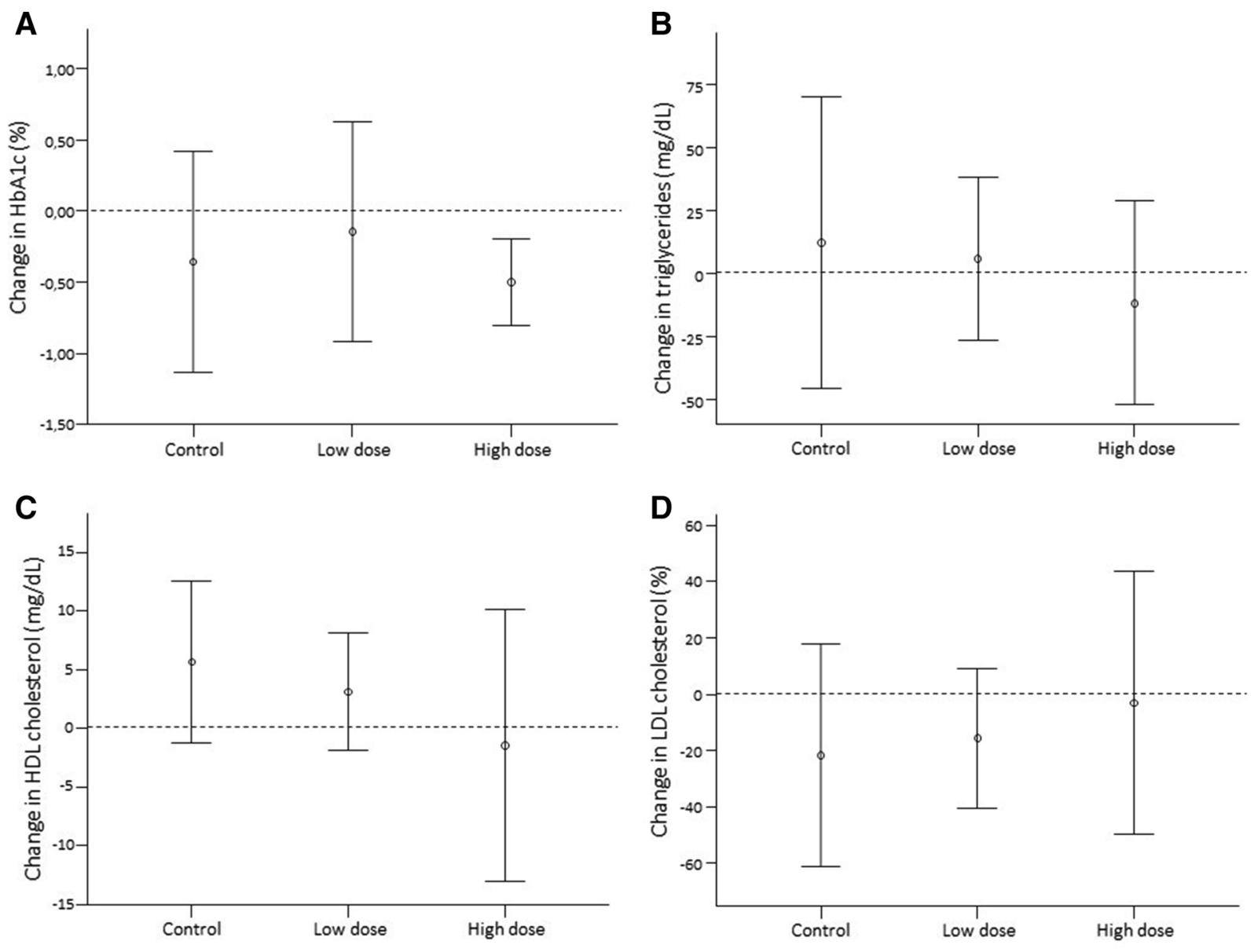

Fig. 3 Change in glycated hemoglobin and lipid risk factors (week 8-week 0) by treatment group. a Change in HbAlc; b change in plasma triglycerides; c change in

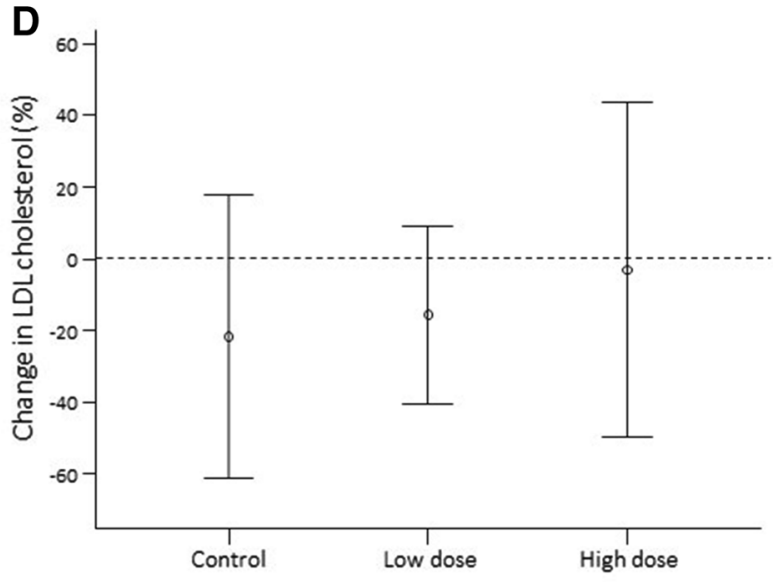

plasma HDL cholesterol; d change in plasma LDL cholesterol. Data are mean \pm SD

and should be suppressed by caloric ingestion. The ability to suppress ghrelin production after a glucose load did not differ systematically across treatment groups (Fig. 5). GLP-1 is produced by intestinal $\mathrm{L}$ cells in response to their contact with nutrients, potentiating insulin secretion and suppressing appetite. There was a trend toward higher post-load GLP-1 concentrations using the study formulation (Fig. 5). FGF-21 is secreted by hepatocytes and muscle cells and induces the differentiation of white adipose tissue to beige or brown adipose tissue. FGF-21 increased in the control group, but decreased slightly in the intervention groups. On the other hand, irisin is secreted by muscle cells under stress or repeated contraction and

also induces the browning of white adipocytes. Plasma irisin decreased in the control group, while it increased in a dose-dependent fashion in the intervention groups (Fig. 6).

\section{Adherence and Adverse Events}

In all study groups, adverse events were mild and rare (Table 3 ) and led to treatment discontinuation in only one study participant from the high-dose group, who disliked the taste of the formulation. Adherence (number of doses actually taken) in the whole study group was 93\% and did not differ among treatment groups (Table 4). 
Table 2 Change in metabolic parameters (week 8 -week 0 ) by intervention group

\begin{tabular}{lccc}
\hline & Control & Low dose & High dose \\
\hline Body weight $(\mathrm{kg})$ & $0.20 \pm 0.75$ & $1.17 \pm 1.43$ & $0.38 \pm 1.51$ \\
Body mass index $\left(\mathrm{kg} / \mathrm{m}^{2}\right)$ & $0.07 \pm 0.28$ & $0.42 \pm 0.58$ & $0.13 \pm 0.61$ \\
Percent body fat & $0.18 \pm 1.57$ & $-0.13 \pm 2.61$ & $0.22 \pm 1.52$ \\
Waist circumference $(\mathrm{cm})$ & $1.74 \pm 2.48$ & $0.48 \pm 4.32$ & $-1.05 \pm 1.05$ \\
Muscle mass $(\mathrm{kg})$ & $-0.30 \pm 1.36$ & $0.60 \pm 1.05$ & $-0.50 \pm 0.74$ \\
Systolic blood pressure $(\mathrm{mmHg})$ & $5.11 \pm 12.89$ & $4.11 \pm 12.02$ & $4.17 \pm 4.12$ \\
Diastolic blood pressure $(\mathrm{mmHg})$ & $4.78 \pm 9.32$ & $4.56 \pm 9.75$ & $0.83 \pm 8.18$ \\
Fasting insulin (microUI/ml) & $0.69 \pm 2.11$ & $2.66 \pm 3.14$ & $1.26 \pm 2.89$ \\
120 min. insulin in OGTT (microUI/ml) & $-6.0 \pm 10.3$ & $-5.3 \pm 23.0$ & $6.6 \pm 33.8$ \\
Incremental AUC-insulin $(\mathrm{microUI} / \mathrm{ml} \times \mathrm{h})$ & $-6.71 \pm 10.89$ & $-7.96 \pm 22.73$ & $5.35 \pm 31.47$ \\
Plasma creatinine (mg/dl) & $-0.09 \pm 0.12$ & $-0.05 \pm 0.13$ & $-0.11 \pm 0.06$ \\
Urinary albumin/creatinine ratio $(\mathrm{mg} / \mathrm{g})$ & $-3.43 \pm 6.0$ & $-0.78 \pm 3.9$ & $-5.03 \pm 10.7$ \\
Post-glucose ghrelin suppression $(\mathrm{pg} / \mathrm{ml})$ & $-37.4 \pm 60.7$ & $40.6 \pm 205.7$ & $-8.3 \pm 86.3$ \\
Post-glucose GLP-1 secretion $(\mathrm{pg} / \mathrm{ml})$ & $-47.8 \pm 178.4$ & $10.3 \pm 70.4$ & $14.6 \pm 72.8$ \\
Plasma FGF-21 (ng/ml) & $0.02 \pm 0.07$ & $-0.08 \pm 0.15$ & $-0.05 \pm 0.07$ \\
Plasma irisin (ng/ml) & $-20.9 \pm 48.8$ & $14.4 \pm 36.9$ & $10.1 \pm 14$ \\
\hline Dan are & &
\end{tabular}

Data are mean $\pm S D$

\section{DISCUSSION}

Using a randomized controlled trial methodology, we performed an initial pilot evaluation of a lipid-based formulation aimed at improving insulin resistance and associated disturbances among apparently healthy adults at risk of T2DM. Despite average fasting plasma glucose and $\mathrm{HbA1c}$ levels considered to be within the normal range, we observed a formulation-induced modulation of whole-body insulin sensitivity over the 8-week duration of the study as well as numeric improvements in glycemic excursions during the OGTT. We also observed favorable changes in several metabolic variables associated with insulin sensitivity, including abdominal obesity and inflammation.

The observed change in insulin sensitivity as reflected by the ISI-Gutt was dose-dependent, reaching $10 \%$ in the high-dose group. The ISIGutt has the great advantage of employing both fasting and post-load glucose and insulin measures to estimate insulin sensitivity overall, not just in one of the two alimentary conditions. Insulin resistance is a major pathogenic factor not just for T2DM [30], but also for cardiovascular disease [6] even in normoglycemic individuals [31]. Opportune intervention to improve insulin sensitivity and glucose tolerance in at-risk individuals holds great potential for impact: A Markov model applied to a simulated population from the US National Health and Nutrition Examination Survey estimated that correcting insulin resistance in young adults could prevent up to $42 \%$ of cardiovascular disease events at the population level [32]. Of note, participants in the low-dose group exhibited an entirely different pattern of postload glycemic excursion, the difference versus the other groups being most pronounced at 60 min post-glucose load. The somewhat puzzling finding of improved glucose tolerance in 

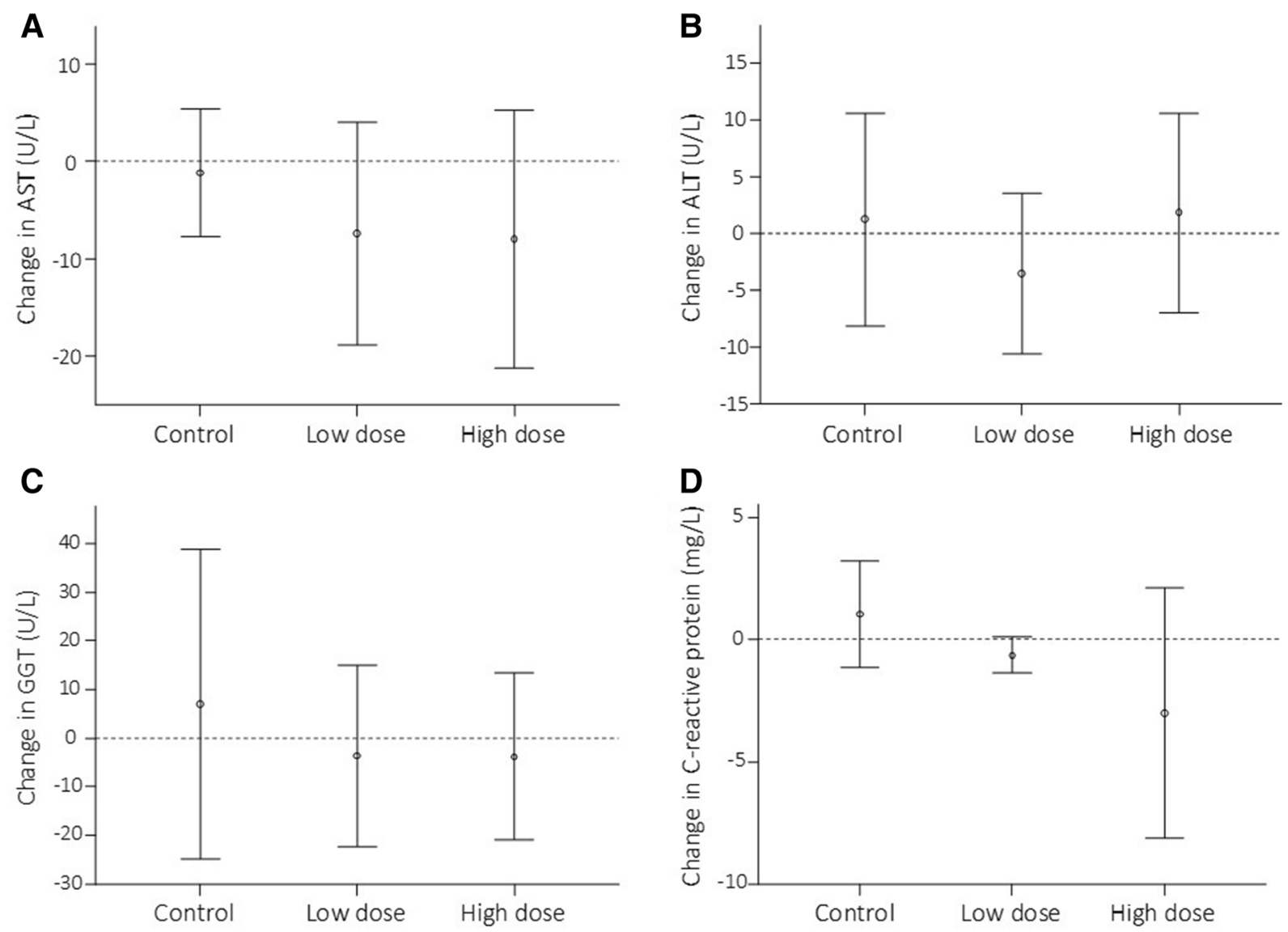

Fig. 4 Change in liver enzymes and C-reactive protein by treatment group. a Change in aspartate amino transferase (AST); b change in alanine amino transferase (ALT);

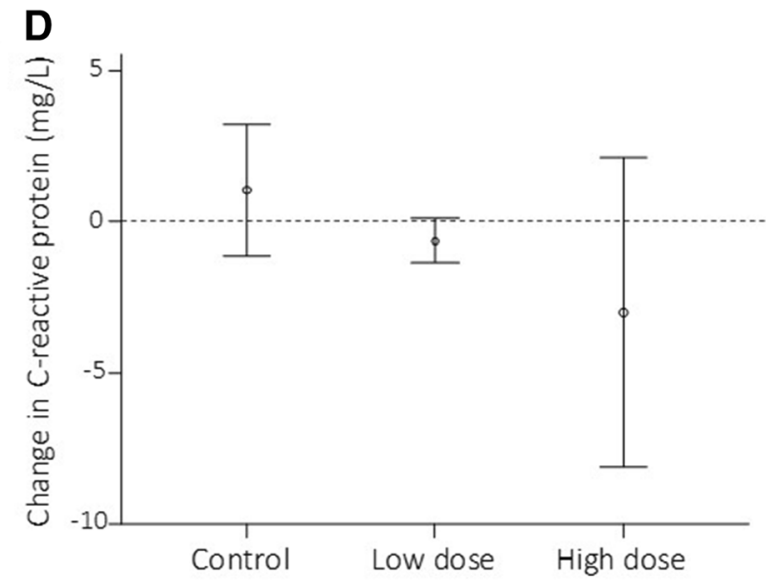

c change in gamma-glutamyl transpeptidase (GGT); d change in C-reactive protein. Data are mean \pm SD

the low-dose, but unchanged glucose tolerance in the high-dose group could be explained by either the existence of an optimal concentration of the components of the study formulation or by some sort of interaction between the study formulation and sunflower oil, the control intervention. We will need further studies to explore this issue in greater detail. We did observe a reduction of $\mathrm{HbA1c}$ in the high-dose group, which did not reach significance. HbA1c reflects average plasma glucose over the last 90-120 days, and the study duration was 8 weeks ( 56 days), so it is possible that the effect was partially diluted by HbA1c levels prior to the study.

Supplementation with the study formulation at high dose also induced a small but significant reduction of waist circumference, indicating

positive changes in body fat distribution. Abdominal obesity is an independent and powerful risk factor for T2DM [33], cardiovascular disease [34] and several types of cancer [35-38], and non-pharmacologic interventions like the study formulation might be a helpful addition to diet and exercise in its management. Abdominal obesity is usually accompanied by a state of chronic, low-degree inflammation that manifests systemically as mild increases in acute-phase reactants such as C-reactive protein $[39,40]$. We found reductions of C-reactive protein in both intervention groups, suggesting that the favorable changes in body fat distribution did translate into reduced systemic inflammation, a major pathogenic factor common to metabolic and cardiovascular diseases [41, 42]. 
A Change in ghrelin suppression (week 8-week 0)

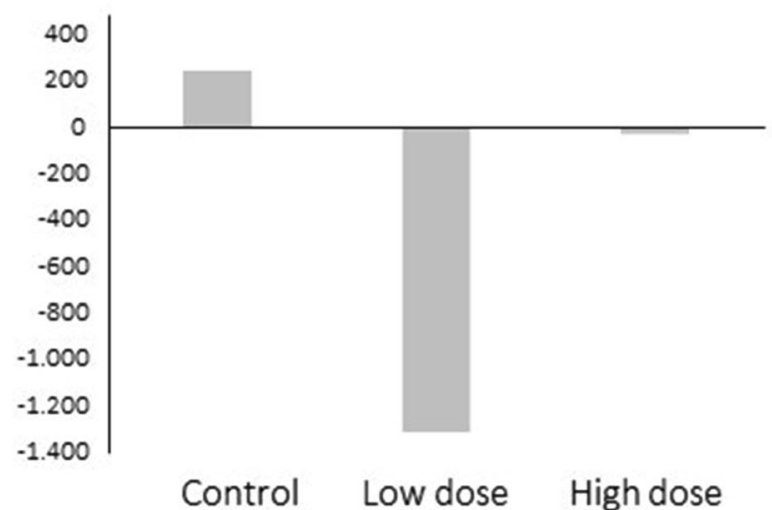

Fig. 5 Changes in gastrointestinal hormone response to a glucose load in OGTT by treatment group. a Mean percent change in suppression of ghrelin secretion
B Change in GLP-1 secretion (week 8 - week 0)

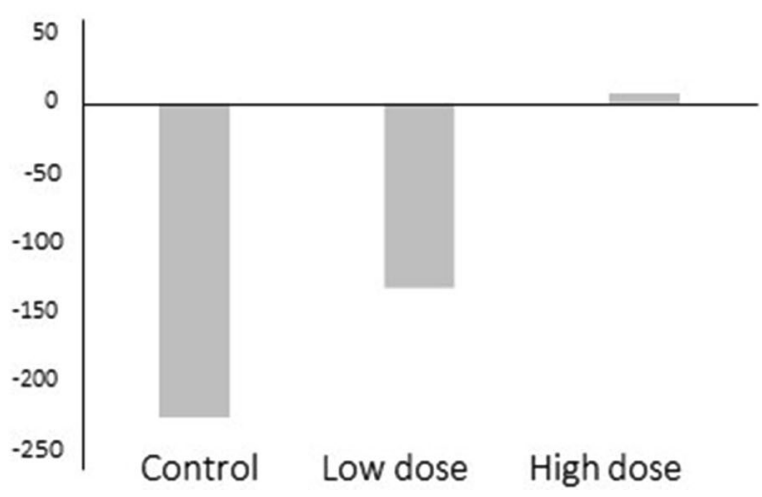

(120 min plasma ghrelin-fasting plasma ghrelin); b mean percent change in secretion of glucagon-like peptide (GLP1) (120 min plasma GLP-1-fasting plasma GLP-1)
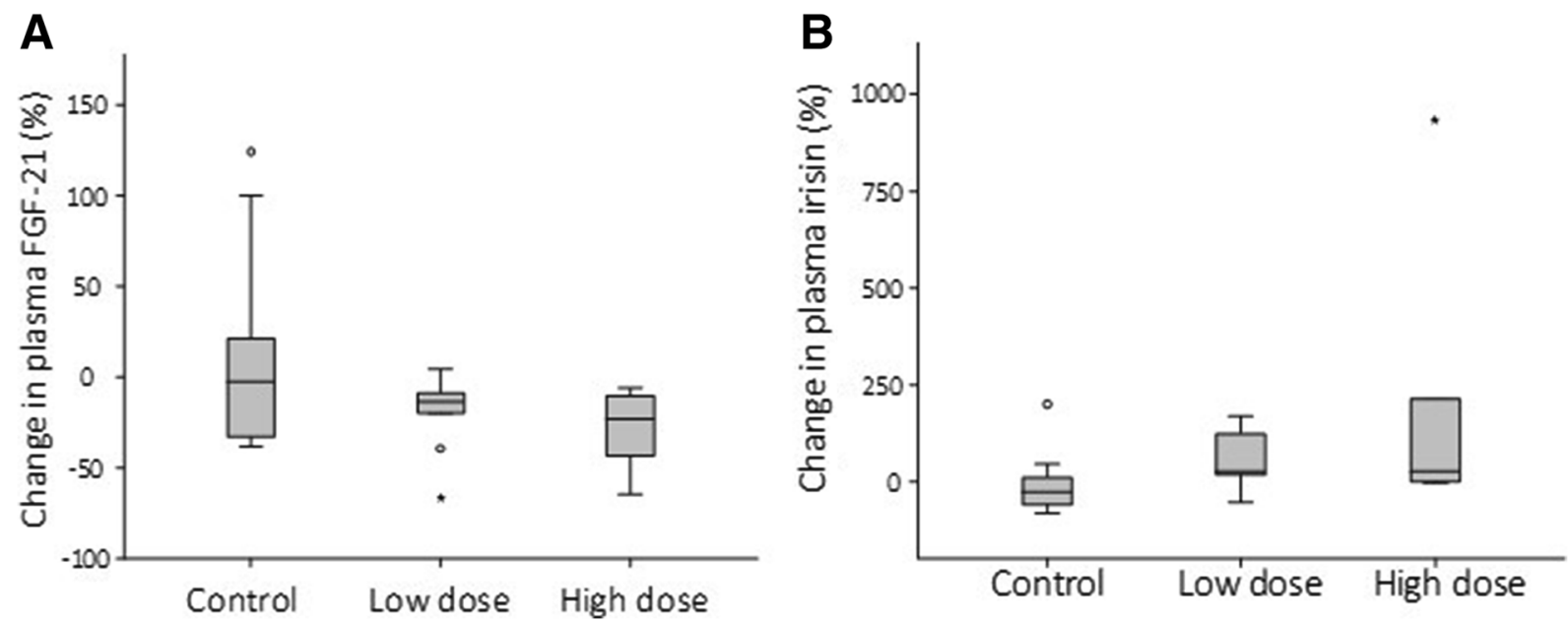

Fig. 6 Box plot of changes in plasma myokines by treatment group. a Percent change in plasma fibroblast growth factor-21 (FGF-21); b percent change in plasma irisin

We observed an interesting dose-response correlation between consumption of the study formulation and GLP-1 secretion. GLP-1 has several favorable metabolic effects [43], but most of them are mediated through modulation of appetite and body weight, which in this study remained unmodified in the three study groups. However, recent evidence suggests that GLP-1 may directly facilitate insulin signaling in adipocytes through alleviation of endoplasmic reticulum stress [44]. This observation related to GLP-1 merits further examination in future studies of the dietary formulation. Another interesting observation was a dose-dependent increase in plasma irisin associated with consumption of the study formulation. While the biology of this recently discovered myokine is only partially understood, irisin is known to induce the expression of uncoupling proteins and a "browning" phenotype in adipose tissue, associated with an increased expression of GLUT4 glucose transporters [45], glucose uptake [46], reduced lipogenesis and increased lipolysis [47]. Similar effects have 
Table 3 Patient-reported adverse events by intervention group

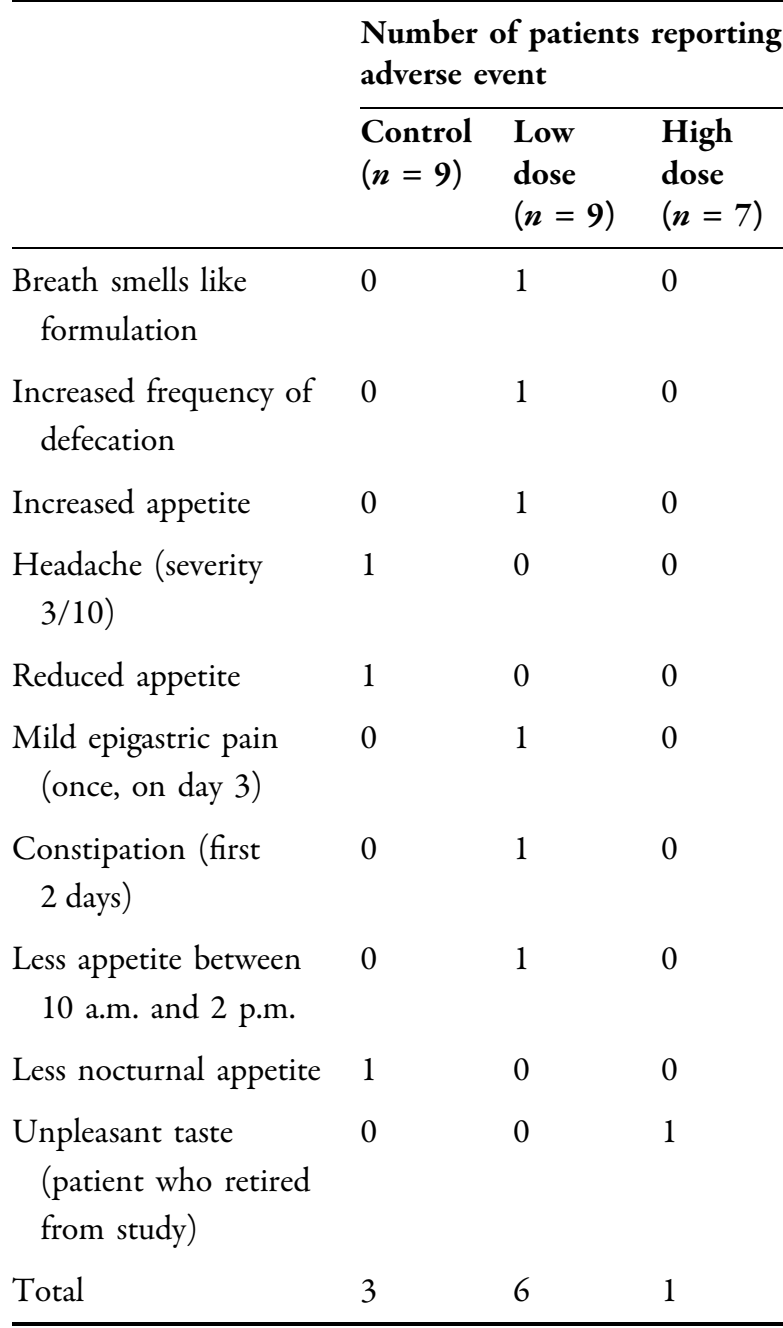

All adverse events were reported between weeks 0 and 8; none were reported between weeks 4 and 8

been reported for irisin on muscle [48] and liver [49] cells. It is plausible that some of the effects observed in the active treatment groups were induced by this increase in circulating irisin, but we cannot positively assert this. Nonetheless, the finding of a clear trend toward increased plasma irisin using our study formulation is encouraging.

The main limitations of the study are its sample size and relatively short 8-week duration. Concerning sample size, this trial was intended as an initial exploration of the effects
Table 4 Patient-reported adherence by intervention group

\begin{tabular}{|c|c|c|c|c|}
\hline \multirow{2}{*}{$\begin{array}{l}\text { Number of doses } \\
\text { not taken }\end{array}$} & \multicolumn{3}{|l|}{ Group } & \multirow[t]{2}{*}{ Total } \\
\hline & Control & $\begin{array}{l}\text { Low } \\
\text { dose }\end{array}$ & $\begin{array}{l}\text { High } \\
\text { dose }\end{array}$ & \\
\hline \multicolumn{5}{|l|}{ Weeks $0-4$} \\
\hline 0 & 3 & 1 & 2 & 6 \\
\hline 1 & 3 & 3 & 0 & 6 \\
\hline 2 & 2 & 2 & 1 & 5 \\
\hline 3 & 1 & 1 & 2 & 4 \\
\hline 4 & 0 & 0 & 1 & 1 \\
\hline 6 & 0 & 1 & 0 & 1 \\
\hline 9 & 0 & 1 & 0 & 1 \\
\hline $\begin{array}{l}\text { Total participants in } \\
\text { group }\end{array}$ & 9 & 9 & 6 & 24 \\
\hline $\begin{array}{l}\text { Average doses } \\
\text { taken/participant }\end{array}$ & 26.9 & 25.2 & 26.0 & 26.1 \\
\hline Average adherence & $96 \%$ & $90 \%$ & $92.8 \%$ & $93 \%$ \\
\hline \multicolumn{5}{|l|}{ Weeks 4-8 } \\
\hline 0 & 4 & 2 & 2 & 8 \\
\hline 1 & 2 & 0 & 1 & 3 \\
\hline 2 & 3 & 1 & 1 & 5 \\
\hline 3 & 0 & 3 & 0 & 3 \\
\hline 4 & 0 & 1 & 2 & 3 \\
\hline 5 & 0 & 1 & 0 & 1 \\
\hline 6 & 0 & 1 & 0 & 1 \\
\hline $\begin{array}{l}\text { Total participants in } \\
\text { group }\end{array}$ & 9 & 9 & 6 & 24 \\
\hline $\begin{array}{l}\text { Average doses } \\
\text { taken/participant }\end{array}$ & 27.1 & 24.7 & 26.2 & 26.0 \\
\hline Average adherence & $96.7 \%$ & $88.2 \%$ & $93.5 \%$ & $92.9 \%$ \\
\hline
\end{tabular}

of the supplementary formulation, but the promising results will merit a deeper exploration in a larger sample size. Regarding study duration, the relevant outcomes (insulin resistance, glucose tolerance, other metabolic 
outcomes) are readily influenced by pharmacologic or non-pharmacologic interventions $[50,51]$, so 8 weeks was deemed an appropriate time frame for any effects of the formulation to be evident.

\section{CONCLUSION}

In conclusion, we undertook an initial evaluation of a lipid-based dietary formulation with a composition aimed at improving insulin resistance and associated derangements in individuals at risk for T2DM. We observed favorable changes in insulin sensitivity, glucose tolerance, body fat distribution and inflammation, but these effects and their durability will need to be assessed more extensively in future studies.

\section{ACKNOWLEDGEMENTS}

We thank all study participants for their valuable patience and collaboration. We thank Ms. Luz D. Nieves-Barreto for her laboratory support. We thank Elkin Darío Rueda and Julio Cesar España from Team Foods Colombia S.A. for their continued support in the preparation of the dietary composition and its chromatographic analyses.

Funding. The study was funded in its entirety by Team Foods Colombia (Grant Number 111-2017). However, the design, execution, analysis, writing and decision to submit rested entirely on the research group, as was established from the beginning in the study contract. No Rapid Service Fee was received by the journal for the publication of this article.

Authorship. All named authors meet the International Committee of Medical Journal Editors (ICMJE) criteria for authorship for this article, take responsibility for the integrity of the work as a whole, and have given their approval for this version to be published.

Disclosures. Luisa F. Gómez-Arango is an employee of Team Foods Colombia. Daniel Colmenares-Araque is an employee of Team
Foods Colombia. Camilo A. Castro-López is an employee of Team Foods Colombia. Eddy Betancourt-Villamizar is an employee of Team Foods Colombia. Jesús Jaimes-Madrigal is an employee of Team Foods Colombia. Carlos A. Alvarez is an employee of Team Foods Colombia. Carlos O. Mendivil has received research funding from Team Foods Colombia, but does not hold stock holdings or options in an organization that may have financial interest in the publication of this manuscript or have any other financial or non-financial competing interests to declare in relation to this manuscript. María J. Peláez-Jaramillo, Natalia Valencia-Enciso, Allison A. Cárdenas-Mojica, Paula V. Gaete, Eitan A. Scher-Nemirovsky, Mario A. Jiménez-Mora, Pedro J. Quiroga-Padilla and Danna K. Puerto-Baracaldo have nothing to disclose.

Compliance with Ethics Guidelines. The study was approved by a legally constituted external IRB (Riesgo de Fractura, SAS), according to minute 30194 of 2018 . All patients underwent an extensive and detailed informed consent procedure and provided written informed consent for their participation. All study procedures were executed in compliance with scientific, technical and administrative regulations for health research dictated by resolution 008430-1993 of the Colombian Ministry of Health and with the principles stated by the Helsinki Declaration of 1964 and its later amendments. The study is registered in the public registry of clinicaltrials.gov under number NCT03512665.

Data Availability. The datasets during and/ or analyzed during the current study are available from the corresponding author on reasonable request.

Open Access. This article is distributed under the terms of the Creative Commons Attribution-NonCommercial 4.0 International License (http://creativecommons.org/licenses/ by-nc/4.0/), which permits any noncommercial use, distribution, and reproduction in any medium, provided you give appropriate credit to the original author(s) and the source, provide 
a link to the Creative Commons license, and indicate if changes were made.

\section{REFERENCES}

1. GBD 2017 Causes of Death Collaborators. Global, regional, and national age-sex-specific mortality for 282 causes of death in 195 countries and territories, 1980-2017: a systematic analysis for the Global Burden of Disease Study 2017. Lancet. 2018;392:1736-88.

2. GBD 2017 Risk Factor Collaborators. Global, regional, and national comparative risk assessment of 84 behavioural, environmental and occupational, and metabolic risks or clusters of risks for 195 countries and territories, 1990-2017: a systematic analysis for the Global Burden of Disease Study 2017. Lancet. 2018;392:1923-94.

3. Faerch K, Borch-Johnsen K, Holst JJ, Vaag A. Pathophysiology and aetiology of impaired fasting glycaemia and impaired glucose tolerance: does it matter for prevention and treatment of type 2 diabetes? Diabetologia. 2009;52:1714-23.

4. Johnson AM, Olefsky JM. The origins and drivers of insulin resistance. Cell. 2013;152:673-84.

5. Buysschaert M, Medina JL, Bergman M, Shah A, Lonier J. Prediabetes and associated disorders. Endocrine. 2015;48:371-93.

6. Laakso M, Kuusisto J. Insulin resistance and hyperglycaemia in cardiovascular disease development. Nat Rev Endocrinol. 2014;10:293-302.

7. Goldstein JN, Long JA, Arevalo D, Ibrahim SA, Mao JJ. US veterans use vitamins and supplements as substitutes for prescription medication. Med Care. 2014;52:S65-9.

8. Bishop FL, Yardley L, Lewith G. Developing a measure of treatment beliefs: the complementary and alternative medicine beliefs inventory. Complement Ther Med. 2005;13:144-9.

9. O'Callaghan FV, Jordan N. Postmodern values, attitudes and the use of complementary medicine. Complement Ther Med. 2003;11:28-32.

10. Shabbir MA, Khan MR, Saeed M, Pasha I, Khalil AA, Siraj N. Punicic acid: a striking health substance to combat metabolic syndromes in humans. Lipids Health Dis. 2017; 16:99.

11. Vroegrijk IOCM, Diepen JAV, Berg SVD, et al. Pomegranate seed oil, a rich source of PA, prevents diet-induced obesity and insulin resistance in mice. Food Chem Toxicol. 2011;49:1426-30.

12. Arao $\mathrm{K}$, Wang $\mathrm{Y}$, Inoue $\mathrm{N}$, et al. Dietary effect of pomegranate seed oil rich in 9cis, 11trans, 13cis conjugated linolenic acid on lipid metabolism in obese, hyperlipidemic OLETF rats. Lipids Health Dis. 2004;3:24-30.

13. Cao H, Gerhold K, Mayers JR, Wiest MM, Watkins SM, Hotamisligil GS. Identification of a lipokine, a lipid hormone linking adipose tissue to systemic metabolism. Cell. 2008;134:933-44.

14. Mozaffarian D, Cao H, King IB, et al. Trans-palmitoleic acid, metabolic risk factors, and new-onset diabetes in US adults: a cohort study. Ann Intern Med. 2010;153:790-9.

15. Shiba S, Tsunoda N, Wakutsu M, et al. Regulation of lipid metabolism by palmitoleate and eicosapentaenoic acid (EPA) in mice fed a high-fat diet. Biosci Biotechnol Biochem. 2011;75:2401-3.

16. Bernstein AM, Roizen MF, Martinez L. Purified palmitoleic acid for the reduction of high-sensitivity C-reactive protein and serum lipids: a doubleblinded, randomized, placebo controlled study. J Clin Lipidol. 2014;8:612-7.

17. Pasman WJ, Heimerikx J, Rubingh CM, et al. The effect of Korean pine nut oil on in vitro CCK release, on appetite sensations and on gut hormones in post-menopausal overweight women. Lipids Health Dis. 2008;7:10.

18. Hua Y, Clark S, Ren J, Sreejayan N. Molecular mechanisms of chromium in alleviating insulin resistance. J Nutr Biochem. 2012;23:313-9.

19. Sharma S, Agrawal RP, Choudhary M, Jain S, Goyal $S$, Agarwal V. Beneficial effect of chromium supplementation on glucose, HbA1C and lipid variables in individuals with newly onset type-2 diabetes. J Trace Elem Med Biol. 2011;25:149-53.

20. Ali A, Ma Y, Reynolds J, Wise JP Sr, Inzucchi SE, Katz DL. Chromium effects on glucose tolerance and insulin sensitivity in persons at risk for diabetes mellitus. Endocr Pract. 2011;17:16-25.

21. Jain SK, Kahlon G, Morehead L, et al. Effect of chromium dinicocysteinate supplementation on circulating levels of insulin, TNF- $\alpha$, oxidative stress, and insulin resistance in type 2 diabetic subjects: randomized, double-blind, placebo-controlled study. Mol Nutr Food Res. 2012;56:1333-41.

22. Ghosh S, Banerjee S, Sil PC. The beneficial role of curcumin on inflammation, diabetes and neurodegenerative disease: a recent update. Food Chem Toxicol. 2015;83:111-24. 
23. Na LX, Li Y, Pan HZ, et al. Curcuminoids exert glucose-lowering effect in type 2 diabetes by decreasing serum free fatty acids: a double-blind, placebo-controlled trial. Mol Nutr Food Res. 2013;57:1569-77.

24. Mohammadi A, Sahebkar A, Iranshahi M, et al. Effects of supplementation with curcuminoids on dyslipidemia in obese patients: a randomized crossover trial. Phytother Res. 2013;27:374-9.

25. Rondanelli M, Monteferrario F, Faliva MA, Perna S, Antoniello N. Key points for maximum effectiveness and safety for cholesterol-lowering properties of plant sterols and use in the treatment of metabolic syndrome. J Sci Food Agric. 2013;93:2605-10.

26. Barengo NC, Tamayo DC, Tono T, Tuomilehto J. A Colombian diabetes risk score for detecting undiagnosed diabetes and impaired glucose regulation. Prim Care Diabetes. 2017;11:86-93.

27. Gutt M, Davis CL, Spitzer SB, et al. Validation of the insulin sensitivity index (ISI $(0,120))$ : comparison with other measures. Diabetes Res Clin Pract. 2000;47:177-84.

28. Monsalve Álvarez JM, González Zapata L. Development of a questionnaire to assess food intake in the University of Antioquia, Colombia. Nutr Hosp. 2011;26:1333-44.

29. Ruiz-Casado A, Alejo LB, Santos-Lozano A, et al. Validity of the physical activity questionnaires IPAQ-SF and GPAQ for cancer survivors: insights from a Spanish cohort. Int $J$ Sports Med. 2016;37:979-85.

30. Meigs JB, Rutter MK, Sullivan LM, Fox CS, D'Agostino RB Sr, Wilson PW. Impact of insulin resistance on risk of type 2 diabetes and cardiovascular disease in people with metabolic syndrome. Diabetes Care. 2007;30:1219-25.

31. Bressler P, Bailey SR, Matsuda M, deFronzo RA. Insulin resistance and coronary artery disease. Diabetologia. 1996;39:1345-50.

32. Eddy D, Schlessinger L, Kahn R, Peskin B, Schiebinger R. Relationship of insulin resistance and related metabolic variables to coronary artery disease: a mathematical analysis. Diabetes Care. 2009;32:361-6.

33. Xue $\mathrm{H}$, Wang $\mathrm{C}, \mathrm{Li} \mathrm{Y}$, et al. Incidence of type 2 diabetes and number of events attributable to abdominal obesity in China: a cohort study. J Diabetes. 2016;8:190-8.

34. Després JP, Lemieux I, Bergeron J, et al. Abdominal obesity and the metabolic syndrome: contribution to global cardiometabolic risk. Arterioscler Thromb Vasc Biol. 2008;28:1039-49.

35. Dong Y, Zhou J, Zhu Y, et al. Abdominal obesity and colorectal cancer risk: systematic review and meta-analysis of prospective studies. Biosci Rep. 2017;37:BSR20170945.

36. Du X, Hidayat K, Shi BM. Abdominal obesity and gastroesophageal cancer risk: systematic review and meta-analysis of prospective studies. Biosci Rep. 2017;11(37):BSR20160474.

37. Hidayat K, Du X, Chen G, Shi M, Shi B. Abdominal obesity and lung cancer risk: systematic review and meta-analysis of prospective studies. Nutrients. 2016;8:E810.

38. Genkinger JM, Kitahara CM, Bernstein L, de Gonzalez BA, Brotzman M, Elena JW. Central adiposity, obesity during early adulthood, and pancreatic cancer mortality in a pooled analysis of cohort studies. Ann Oncol. 2015;26:2257-66.

39. Santos AC, Lopes C, Guimarães JT, Barros H. Central obesity as a major determinant of increased high-sensitivity C-reactive protein in metabolic syndrome. Int J Obes (Lond). 2005;29:1452-6.

40. Bennett NR, Ferguson TS, Bennett FI, et al. Highsensitivity C-reactive protein is related to central obesity and the number of metabolic syndrome components in Jamaican young adults. Front Cardiovasc Med. 2014;1:12.

41. Hotamisligil GS. Inflammation, metaflammation and immunometabolic disorders. Nature. 2017;542:177-85.

42. Lopez-Candales A, Hernández Burgos PM, Hernandez-Suarez DF, Harris D. Linking chronic inflammation with cardiovascular disease: from normal aging to the metabolic syndrome. J Nat Sci. 2017;3:e341.

43. Sandoval DA, D'Alessio DA. Physiology of proglucagon peptides: role of glucagon and GLP-1 in health and disease. Physiol Rev. 2015;95:513-48.

44. Jiang Y, Wang Z, Ma B, et al. GLP-1 improves adipocyte insulin sensitivity following induction of endoplasmic reticulum stress. Front Pharmacol. 2018;9:1168.

45. Boström P, Wu J, Jedrychowski MP, et al. A PGC1- $\alpha$ dependent myokine that drives brown-fat-like development of white fat and thermogenesis. Nature. $2012 ; 481: 463-8$.

46. Huh JY, Dincer F, Mesfum E, Mantzoros CS. Irisin stimulates muscle growth-related genes and 
regulates adipocyte differentiation and metabolism in humans. Int J Obes. 2014;38:1538-44.

47. Xiong XQ, Chen D, Sun HJ, et al. FNDC5 overexpression and irisin ameliorate glucose/lipid metabolic derangements and enhance lipolysis in obesity. Biochim Biophys Acta. 2015;852:1867-75.

48. Vaughan RA, Gannon NP, Barberena MA, et al. Characterization of the metabolic effects of irisin on skeletal muscle in vitro. Diabetes Obes Metab. 2014;16:711-8.

49. Mo L, Shen J, Liu Q, et al. Irisin is regulated by CAR in liver and is a mediator of hepatic glucose and lipid metabolism. Mol Endocrinol. 2016;30:533-42.
50. Motahari-Tabari N, Ahmad Shirvani M, Shirzad-EAhoodashty M, Yousefi-Abdolmaleki E, Teimourzadeh M. The effect of 8 weeks aerobic exercise on insulin resistance in type 2 diabetes: a randomized clinical trial. Glob J Health Sci. 2014;7:115-21.

51. Madsen SM, Thorup AC, Overgaard K, Jeppesen PB. High intensity interval training improves glycaemic control and pancreatic $\beta$ cell function of type 2 diabetes patients. PLoS One. 2015;10:e0133286. 